PROCEEDINGS OF THE

AMERICAN MATHEMATICAL SOCIETY

Volume 126, Number 11, November 1998, Pages 3283-3292

S $0002-9939(98) 04402-5$

\title{
POLYNOMIAL APPROXIMATION WITH VARYING WEIGHTS ON COMPACT SETS OF THE COMPLEX PLANE
}

\author{
IGOR E. PRITSKER
}

(Communicated by Theodore W. Gamelin)

\begin{abstract}
For a compact set $E$ with connected complement, let $A(E)$ be the uniform algebra of functions continuous on $E$ and analytic interior to $E$. We describe $A(E, W)$, the set of uniform limits on $E$ of sequences of the weighted polynomials $\left\{W^{n}(z) P_{n}(z)\right\}_{n=0}^{\infty}$, as $n \rightarrow \infty$, where $W \in A(E)$ is a nonvanishing weight on $E$. If $E$ has empty interior, then $A(E, W)$ is completely characterized by a zero set $Z_{W} \subset E$. However, if $E$ is a closure of Jordan domain, the description of $A(E, W)$ also involves an inner function.

In both cases, we exhibit the role of the support of a certain extremal measure, which is the solution of a weighted logarithmic energy problem, played in the descriptions of $A(E, W)$.
\end{abstract}

\section{INTRODUCTION}

Let $E$ be a compact set in the complex plane $\mathbb{C}$ with the connected complement $\overline{\mathbb{C}} \backslash E$. We denote the uniform algebra of continuous on $E$ and analytic in the interior of $E$ functions by $A(E)$ (see, e.g., [5, p. 25]). Clearly, the corresponding uniform norm for any $f \in A(E)$ is defined by

$$
\|f\|_{E}:=\max _{z \in E}|f(z)| .
$$

Consider a weight function $W \in A(E)$ such that $W(z) \neq 0$ for any $z \in E$, and define the weighted polynomials $W^{n}(z) P_{n}(z)$, where $P_{n}(z)$ is an algebraic polynomial in $z$ with complex coefficients, with $\operatorname{deg} P_{n} \leq n$. Note that the power of weight varies with the degree of polynomial. We are interested in a description of the function set $A(E, W)$, consisting of the uniform limits on $E$ of sequences of the weighted polynomials $\left\{W^{n}(z) P_{n}(z)\right\}_{n=0}^{\infty}$, as $n \rightarrow \infty$. It is well known that if $W(z) \equiv 1$ on $E$ then $A(E, 1)=A(E)$ by Mergelyan's theorem [5, p. 48]. In general, we have that $A(E, W) \subset A(E)$.

Our problem originated in the work of Lorentz [12] on incomplete polynomials on the real line. Surveys of results in this area, dealing with weighted approximation on the real line, can be found in [22] and $[18, \mathrm{Ch}$. VI]. The most recent developments are in $[8]-[10]$.

Received by the editors September 4, 1996 and, in revised form, March 25, 1997.

1991 Mathematics Subject Classification. Primary 30E10; Secondary 30B60, 31A15, 41A30.

Key words and phrases. Weighted polynomials, closed ideals, weighted energy problem, logarithmic potentials, uniform algebras.

(C)1998 American Mathematical Society 
The questions of density of the weighted polynomials in the set of analytic functions in a domain have been considered in [4], [15] and [16]. In particular, [16] contains a necessary and sufficient condition such that any analytic in a bounded open set function is uniformly approximable by the weighted polynomials $W^{n}(z) P_{n}(z)$ on compact subsets. However, the description of $A(E, W)$ seems to be much more complicated, in that no general necessary and sufficient condition is known (in terms of the weight $W(z)$ ), even for the real interval case, i.e., for $E=[a, b] \subset \mathbb{R}$.

We shall approach the above mentioned problems on $A(E, W)$, using ideas of the theories of uniform algebras and of weighted potentials.

The second section of this paper deals with certain general inclusions of $A(E, W)$ and some weighted potential background. We state the results on $A(E, W)$ for $E$ having empty interior in Section 3. The corresponding results for $E$ being the closure of the unit disk $D$ or, more generally, of a Jordan domain $G$, are developed in Section 4. Section 5 contains all proofs. We conclude with remarks and open problems in Section 6 .

\section{InClusion of $A(E, W)$ AS a Closed ideal ANd Weighted potentials}

We start with

Proposition 2.1. $A(E, W)$, endowed with norm (1.1), is a closed function algebra (not necessarily containing constants and separating points).

We have already remarked that $A(E, W) \subset A(E)$. To make this inclusion more precise, let us introduce the algebra $[W(z), z W(z)]$ generated by the two functions $W(z)$ and $z W(z)$, which is the uniform closure of all polynomials in $W(z)$ and $z W(z)$ (with constant terms included) on $E$. Clearly, $[W(z), z W(z)] \subset$ $A(E)$. Furthermore, since any weighted polynomial $W^{n}(z) P_{n}(z)$ is an element of $[W(z), z W(z)]$, then $A(E, W) \subset[W(z), z W(z)]$. Thus, we arrive at the following

Proposition 2.2. $A(E, W) \subset[W(z), z W(z)] \subset A(E)$.

The next fact is rather simple but important.

Proposition 2.3. $A(E, W)$ is a closed ideal of $[W(z), z W(z)]$.

It turns out that in many cases $[W(z), z W(z)]=A(E)$, so that $A(E, W)$ becomes a closed ideal of $A(E)$ by Proposition 2.3. This situation is the most interesting for us, because then we can employ characterizations of the closed ideals of $A(E)$ for some types of the compact $E$. This is done in Sections 3 and 4.

Proposition 2.4. $[W(z), z W(z)]=A(E)$ iff $1 / W(z) \in[W(z), z W(z)]$.

Unfortunately, we do not know any effectively verifiable necessary and sufficient condition on the weight $W(z)$, so that the equality $[W(z), z W(z)]=A(E)$ is valid. Nevertheless, a number of sufficient conditions can be given, guaranteeing that the two algebras $[W(z), z W(z)]$ and $A(E)$ coincide.

Proposition 2.5. Each of the following conditions implies that $[W(z), z W(z)]=$ $A(E)$ :

(a) The point $\zeta=0$ belongs to the unbounded component of $\overline{\mathbb{C}} \backslash W(E)$;

(b) $E$ is the closure of a Jordan domain or a Jordan arc, and $W(z)$ is one-to-one on $E$;

(c) $E$ is a Jordan arc and $W(z)$ is of bounded variation on $E$; 
(d) $E$ is a Jordan arc and $W(z)$ is locally one-to-one on $E$;

(e) $E=\bar{G}$, where $G$ is a Jordan domain bounded by an analytic curve, and $W^{\prime}(z) \in A(\bar{G})$.

Other sufficient conditions, implying the conclusion of Proposition 2.5, can be found in [1], [6], [21, §30] and [20]. Obviously, if the interior of $E$ is empty, then $A(E)=C(E)$ by definition, where $C(E)$ is the algebra of all continuous functions on $E$.

A very important tool for analyzing the behavior of the weighted polynomials $W^{n} P_{n}$ is the theory of logarithmic potentials with external fields (cf. [18]). Assuming that $E$ has positive logarithmic capacity (cf. [23, p. 55]), then

$$
w(z):= \begin{cases}|W(z)|, & z \in E, \\ 0, & z \notin E,\end{cases}
$$

is an admissible weight for the weighted logarithmic energy problem on $E$ considered in Section I.1 of [18]. This enables us to use certain results of [18], which we summarize below for the convenience of the reader. Recall that the logarithmic potential of a compactly supported Borel measure $\mu$ is given by (cf. [23, p. 53])

$$
U^{\mu}(z):=\int \log \frac{1}{|z-t|} d \mu(t) .
$$

Proposition 2.6. There exists a positive unit Borel measure $\mu_{w}$, with support $S_{w}:=\operatorname{supp} \mu_{w} \subset \partial E$, such that for any polynomial $P_{n}(z), \operatorname{deg} P_{n} \leq n$, we have

$$
\left|W^{n}(z) P_{n}(z)\right| \leq\left\|W^{n} P_{n}\right\|_{S_{w}} \exp \left(n\left(F_{w}-U^{\mu_{w}}(z)+\log |W(z)|\right)\right), \quad z \in E,
$$

where $F_{w}$ is a constant.

Furthermore, the inequality

$$
U^{\mu_{w}}(z)-\log |W(z)| \geq F_{w}
$$

holds quasi-everywhere on $E$, and

$$
U^{\mu_{w}}(z)-\log |W(z)| \leq F_{w}, \text { for any } z \in S_{w} .
$$

By saying quasi-everywhere (q.e.), we mean that a property holds everywhere, with the exception of a set of zero logarithmic capacity. The measure $\mu_{w}$ is the solution of a weighted energy problem, corresponding to the weight $w(z)$ of $(2.1)$ (see Section I.1 of [18]).

It follows from (2.3) and (2.4) that the norm of a weighted polynomial $W^{n} P_{n}$ essentially "lives" on $S_{w}$, i.e.,

$$
\left|W^{n}(z) P_{n}(z)\right| \leq\left\|W^{n} P_{n}\right\|_{S_{w}}
$$

for quasi-every $z \in E$. We can replace, in fact, the words "quasi-everywhere on $E$ " in (2.4) and (2.6) by "everywhere on $E$ ", by requiring a certain regularity for the set $E$. In particular, the following is valid (see Corollary III.2.6 of [18]).

Proposition 2.7. Suppose that for every point $z_{0} \in E$, the set $\left\{z:\left|z-z_{0}\right|<\right.$ $\delta, z \in E\}$ has positive capacity for any $\delta>0$. Then

$$
\left\|W^{n} P_{n}\right\|_{E}=\left\|W^{n} P_{n}\right\|_{S_{w}}
$$

for any polynomial $P_{n}, \operatorname{deg} P_{n} \leq n$.

Consequently, the Shilov boundary (cf. [21, pp. 36-39]) of $A(E, W)$ is contained in $S_{w}$. 
One may consult Section III.2 of [18] on further details, regarding the supremum norm of weighted polynomials.

\section{WeIGHTED APPROXIMATION ON SETS WITH EMPTY INTERIOR}

Let $E$ be a compact set with connected complement and empty interior. Obviously, $A(E)=C(E)$ in this case. We characterize $A(E, W)$ in terms of a certain zero set.

Theorem 3.1. Suppose that $E$ has a connected complement and an empty interior, and that $W \in C(E)$ is a nonvanishing weight on $E$. Assume that $[W(z), z W(z)]=$ $C(E)$.

Then, there exists a closed set $Z_{W} \subset E$ such that

$$
f \in A(E, W) \text { if and only if } f \in C(E) \text { and }\left.f\right|_{Z_{W}} \equiv 0 .
$$

It is clear that $A(E, W)=C(E)$ if and only if the set $Z_{W}$ is empty. This is true, for example, for $W(z) \equiv 1$ on $E$.

Theorem 3.1 generalizes a recent result of Kuijlaars (see Theorem 3 of [10]), related to polynomial approximation with varying weights on the real line. However, it has a new part even in the latter case, allowing us to consider the complex valued weights $W(z)$ on subsets of the real line.

A description of the set $Z_{W}$ in terms of the weight $W(z)$ is unknown in general. We can only show that $Z_{W}$ must contain the complement of $S_{w}$ (see Proposition 2.6) in $E$.

Theorem 3.2. Let $E$ be an arbitrary compact set with the connected complement $\overline{\mathbb{C}} \backslash E$ and let $W \in A(E)$ be a nonvanishing weight on $E$. Suppose that for every point $z_{0} \in E$, the set $\left\{z:\left|z-z_{0}\right|<\delta, z \in E\right\}$ has positive logarithmic capacity for any $\delta>0$. Assume further that $\overline{\mathbb{C}} \backslash S_{w}$ is connected and $[W(z), z W(z)]=C\left(S_{w}\right)$ on $S_{w}$.

If $f \in A(E, W)$, then $f(z)=0$ for any $z \in \overline{E \backslash S_{w}}$. In particular, if $E$ has empty interior, then $\overline{E \backslash S_{w}} \subset Z_{W}$.

The proof of Theorem 3.2 is based on an idea of Kuijlaars (see Theorem 2 and its proof in [10]).

If $E$ is a compact subset of the real line and the weight $W(z)$ is real valued, then condition (a) of Proposition 2.5 is clearly satisfied, so that $[W(z), z W(z)]=$ $C(E)$. Therefore, the conclusion of Theorem 3.1 is valid, and coincides with that of Theorem 3 of [10]. Furthermore, if for any point in $E$, the intersection of its arbitrary neighborhood with $E$ has positive logarithmic capacity, then $\overline{E \backslash S_{w}} \subset Z_{W}$. Since $[W(z), z W(z)]=C\left(S_{w}\right)$ on $S_{w}$ by Proposition 2.5(a), Theorem 3.2 essentially reduces to Theorem 2 of [10] in this case, which in turn contains an earlier result of Theorem 4.1 of [22].

We mention two examples here just for illustrative purposes. A number of additional examples, with their complete discussions, is in [22], [18, Ch. VI], and [8]-[9].

Example 3.3 (Incomplete Polynomials). Let $E=[0,1]$ and $W(x)=x^{\theta /(1-\theta)}$, where $0<\theta<1$. It is known that $S_{w}=\left[\theta^{2}, 1\right]$ and that $A\left([0,1], x^{\theta /(1-\theta)}\right)$ consists of all continuous functions on $[0,1]$, vanishing on $\left[0, \theta^{2}\right]$ (see $[19]$ and $[18$, Sect. VI.1]). Thus, the set $Z_{W}$ of Theorem 3.1 is just $\left[0, \theta^{2}\right]=\overline{E \backslash S_{w}}$ in this case.

However, it is not always true that $Z_{W}=\overline{E \backslash S_{w}}$, as the next example shows. 
Example 3.4 (Exponential Weights). Let $E=[-2,2]$ and $W(x)=\exp \left(-\gamma_{\alpha}|x|^{\alpha}\right)$, where $\alpha>0$ and

$$
\gamma_{\alpha}=\frac{\Gamma(\alpha / 2) \Gamma(1 / 2)}{2 \Gamma(\alpha / 2+1 / 2)}
$$

It is known that $S_{w}=[-1,1]$ and that

$$
\begin{aligned}
& A\left([-2,2], \exp \left(-\gamma_{\alpha}|x|^{\alpha}\right)\right)= \\
& \begin{cases}\left\{f \in C([-2,2]):\left.f\right|_{[-2,-1] \cup[1,2]} \equiv 0\right\} & \text { for } \alpha \geq 1, \\
\left\{f \in C([-2,2]):\left.f\right|_{[-2,-1] \cup[1,2] \cup\{0\}} \equiv 0\right\} & \text { for } 0<\alpha<1,\end{cases}
\end{aligned}
$$

where the range $\alpha>1$ was studied in [13], and the case of the remaining interval $0<\alpha \leq 1$ was covered by the results of [14]. In our context, (3.1) means that $Z_{W}=[-2,-1] \cup[1,2]=\overline{E \backslash S_{w}}$ for $\alpha \geq 1$ and that $Z_{W}=[-2,-1] \cup[1,2] \cup\{0\}=$ $\overline{E \backslash S_{w}} \cup\{0\}$ for $0<\alpha<1$.

\section{Weighted approximation ON THE UNit DisK AND ON JORDAN DOMAINS}

The first result of this section is a consequence of the well-known description of closed ideals of $A(\bar{D})$, where $D$ is the unit disk, due to Beurling (unpublished) and Rudin [17] (see also [7, pp. 82-87] for a discussion). Recall that $g$ is an inner function if it is analytic in $D$, with $\|g\|_{\bar{D}} \leq 1$, and $\left|g\left(e^{i \theta}\right)\right|=1$ almost everywhere on the unit circle (cf. [7, p. 62]). By the factorization theorem, every inner function can be uniquely expressed in the form

$$
g(z)=B(z) S(z), \quad z \in D,
$$

where $B(z)$ is a Blaschke product and $S(z)$ is a singular function, i.e.,

$$
S(z):=\exp \left(-\int \frac{e^{i \theta}+z}{e^{i \theta}-z} d \nu_{s}(\theta)\right), \quad z \in D,
$$

with $\nu_{s}$ being a positive measure on the unit circle, singular with respect to $d \theta$ (see [7, pp. 63-67]).

Theorem 4.1. Let a nonvanishing weight $W \in A(\bar{D})$ be such that $[W(z), z W(z)]$ $=A(\bar{D})$. Assume that $A(\bar{D}, W)$ contains a not identically zero function.

Then there exist a closed set $H_{W} \subset \partial D$ of Lebesgue measure zero and an inner function $g_{W}$ satisfying

(i) every accumulation point of the zeros of its Blaschke product is in $H_{W}$,

(ii) the measure $\nu_{s}$ of its singular function is supported on $H_{W}$; such that

$f \in A(\bar{D}, W)$ if and only if $f=g_{W} h$, where $h \in A(\bar{D})$ and $\left.h\right|_{H_{W}} \equiv 0$.

The case of a Jordan domain $G$ can be reduced to that of the unit disk, using a canonical conformal mapping $\phi: G \rightarrow D$ and its inverse $\psi:=\phi^{-1}$. We state the corresponding result for completeness.

Theorem 4.2. Let a nonvanishing weight $W \in A(\bar{G})$, where $G$ is a Jordan domain, be such that $[W(z), z W(z)]=A(\bar{G})$. Assume that $A(\bar{G}, W)$ contains a not identically zero function. 
Then there exist a closed set $H_{W} \subset \partial D$ and an inner function $g_{W}$, as in Theorem 4.1, such that

where $h \in A(\bar{G})$ and $\left.h\right|_{\psi\left(H_{W}\right)} \equiv 0$.

$$
f \in A(\bar{G}, W) \text { if and only if } f=\left(g_{W} \circ \phi\right) h,
$$

It follows from Theorems 4.1 and 4.2 that $A(\bar{D}, W)=A(\bar{D})$ or $A(\bar{G}, W)=A(\bar{G})$ if and only if $g_{W} \equiv 1$ and $H_{W}$ is empty.

Example 4.3 (Exponential weight on the Szegö domain). Let $W(z)=e^{-z}$ and $G$ be the Szegö domain

$$
\left\{z:\left|z e^{1-z}\right|<1 \text { and }|z|<1\right\},
$$

which is bounded by a piecewise analytic curve (the Szegö curve) with the only corner point $z=1$ (see [15] for more information and the graph of $G$ ). We remark that the function $\phi(z)=z e^{1-z}$ maps $G$ conformally onto $D$. It follows from Proposition 3.1 of [15] that $f_{0}(z):=(z-1) e^{-z}$ belongs to $A\left(\bar{G}, e^{-z}\right)$. Thus, either $H_{W}=\{1\}$ or $H_{W}$ is empty. Clearly, $g_{W} \equiv 1$ and we obtain from Theorem 4.2 that if $f \in A(\bar{G})$ and $f(1)=0$, then $f \in A\left(\bar{G}, e^{-z}\right)$. This implies the result of Theorem 3.2 of [15], in particular. It was also conjectured in [15] that $H_{W}=\{1\}$ (in the present notation), but this problem remains open.

Our next goal is to exhibit the role of the set $S_{w}$ (see Proposition 2.6) in the case of weighted approximation on Jordan domains. Since $W \in A(\bar{G})$ is analytic in $G$, then $S_{w} \subset \partial G$ by Theorem IV.1.10(a) of [18] and (2.1). The following result shows that $S_{w}=\partial G$ is necessary for nontrivial weighted approximation on $\bar{G}$.

Theorem 4.4. Let $G$ be a Jordan domain and let $W \in A(\bar{G})$ be a nonvanishing weight. Assume that $S_{w}$ is a proper subset of $\partial G$ and that $[W(z), z W(z)]=C\left(S_{w}\right)$ on $S_{w}$. Then $A(\bar{G}, W)$ contains the identically zero function only.

We construct below a specific example of the weight $W(z)$, satisfying the conditions of Theorem 4.4.

Example 4.5. Let $G$ be a Jordan domain, as before, and let $\gamma$ be a proper closed subarc of $\partial G$. Define the conformal mapping $\Phi: \overline{\mathbb{C}} \backslash \gamma \rightarrow\{w:|w|>1\}$, normalized by $\Phi(\infty)=\infty$ and $\lim _{z \rightarrow \infty} \Phi(z) / z>0$. We extend $\Phi$ to $\gamma$, using boundary limits from inside of $G$, so that $\Phi \in A(\bar{G})$ is one-to-one on $\bar{G}$. Thus, we can consider approximation by weighted polynomials on $\bar{G}$, with $W(z)=1 / \Phi(z), z \in \bar{G}$.

Denote the Green function of the domain $\overline{\mathbb{C}} \backslash \gamma$, with pole at $\infty$, by $g(z, \infty)[23$, p. 14], and the classical equilibrium distribution of $\gamma$ (in the sense of logarithmic potential theory) by $\mu_{\gamma}[23$, p. 55]. Then,

$$
g(z, \infty)=\log \frac{1}{\operatorname{cap}(\gamma)}-U^{\mu_{\gamma}}(z), \quad z \in \mathbb{C},
$$

where $\operatorname{cap}(\gamma)$ is the logarithmic capacity of $\gamma$ (see [18, Sect. I.4]). Furthermore, since $g(z, \infty)=\log |\Phi(z)|, z \in \mathbb{C}$ (cf. [23, p. 18]), we obtain

$$
U^{\mu_{\gamma}}(z)-\log |1 / \Phi(z)|=\log \frac{1}{\operatorname{cap}(\gamma)}, \quad z \in \mathbb{C} .
$$

It follows from (4.4) and Theorem I.3.3 of [18] that $\mu_{\gamma}$ is the solution of a weighted energy problem (cf. [18, Sec. I.1]) for the weight $w(z)=1 /|\Phi(z)|$ on $\bar{G}$. Hence, we have shown that $S_{w}=\operatorname{supp} \mu_{\gamma}=\gamma$. 
Observe that the weighted polynomials $W^{n}(z) P_{n}(z)$, with $W(z)=1 / \Phi(z), z \in$ $\bar{G}$, can approximate an arbitrary analytic function in $G$ on compact subsets of $G$ by Theorem 1.1 of [16] and (4.4). On the other hand, Proposition 2.5(b) and Theorem 4.4 indicates that they can only approximate the identically zero function uniformly on $\bar{G}$.

\section{Proofs}

Proof of Proposition 2.1. We have to show that $A(E, W)$ is closed under addition, multiplication by constants and by functions of $A(E, W)$, and under uniform limits. Suppose that $W^{n} P_{n} \rightarrow f \in A(E, W)$ and $W^{n} Q_{n} \rightarrow g \in A(E, W)$ uniformly on $E$, as $n \rightarrow \infty$. Then $W^{n}\left(P_{n}+Q_{n}\right) \rightarrow(f+g)$, as $n \rightarrow \infty$, so that $(f+g) \in A(E, W)$. If $\alpha \in \mathbb{C}$ then $W^{n} \alpha P_{n} \rightarrow \alpha f$, as $n \rightarrow \infty$, and $\alpha f \in A(E, W)$. Observe that

$$
\begin{aligned}
& \left\|f g-W^{2 n} P_{n} Q_{n}\right\|_{E} \leq\left\|f g-f W^{n} Q_{n}\right\|_{E}+\left\|f W^{n} Q_{n}-W^{2 n} P_{n} Q_{n}\right\|_{E} \leq \\
& \|f\|_{E}\left\|g-W^{n} Q_{n}\right\|_{E}+\left\|W^{n} Q_{n}\right\|_{E}\left\|f-W^{n} P_{n}\right\|_{E} \rightarrow 0,
\end{aligned}
$$

as $n \rightarrow \infty$, i.e., $f g \in A(E, W)$. Applying the standard diagonalization argument, we see that $A(E, W)$ is closed in norm (1.1).

Proof of Proposition 2.3. Assume that $f \in A(E, W)$ and $W^{n} P_{n} \rightarrow f$ uniformly on $E$, as $n \rightarrow \infty$. Then, for any pair of nonnegative integers $k$ and $\ell$ such that $k \geq \ell$, we have

$$
\begin{aligned}
& \left\|f(z) W^{k}(z) z^{\ell}-W^{n+k}(z) z^{\ell} P_{n}(z)\right\|_{E} \leq \\
& \left\|W^{k}(z) z^{\ell}\right\|_{E}\left\|f-W^{n} P_{n}\right\|_{E} \rightarrow 0, \quad \text { as } n \rightarrow \infty,
\end{aligned}
$$

which gives that $f(z) W^{k}(z) z^{\ell} \in A(E, W)$. Since $A(E, W)$ is closed under addition and multiplication by constants (by Proposition 2.1), then the product of $f$ and any polynomial in $W(z)$ and $z W(z)$ belongs to $A(E, W)$. Thus, if $g \in[W(z), z W(z)]$ then $f g \in A(E, W)$ follows immediately, because $A(E, W)$ is closed in the uniform norm on $E$ (cf. Proposition 2.1). The proof is now complete in view of Propositions 2.1 and 2.2 .

Proof of Proposition 2.4. Obviously, if $[W(z), z W(z)]=A(E)$ then $1 / W(z) \in A(E)$ $=[W(z), z W(z)]$.

Assume that $1 / W(z) \in[W(z), z W(z)]$. It follows that $z \in[W(z), z W(z)]$ and, consequently, every polynomial in $z$ is in $[W(z), z W(z)]$. Since $[W(z), z W(z)]$ is uniformly closed on $E$ by definition, then $A(E) \subset[W(z), z W(z)]$ by Mergelyan's theorem [5, p. 48]. Thus, Proposition 2.2 implies at once that $A(E)=[W(z), z W(z)]$.

Proof of Proposition 2.5. First, we remark that $W(z)$ and $z W(z)$ together separate points of any set $E$.

(a) Observe that $W(E)$, the image of $E$ in $\zeta$-plane under the mapping $\zeta=W(z)$, is compact. By assumption, function $1 / \zeta$ is analytic on the polynomial convex hull of $W(E)$ and can be uniformly approximated there by polynomials in $\zeta$ (by Mergelyan's theorem). Returning to $z$-plane, we obtain that $1 / W(z)$ is uniformly approximable on $E$ by polynomials in $W(z)$. It follows that $[W(z), z W(z)]=A(E)$ by Proposition 2.4 .

(b) The mapping $\zeta=W(z)$ can be continued as a homeomorphism between $z$-plane and $\zeta$-plane (cf. [11, p. 535]). Since $W(z)$ doesn't vanish on $E$, then $\zeta=0$ belongs to the domain $\overline{\mathbb{C}} \backslash W(E)=W(\overline{\mathbb{C}} \backslash E)$, which contains $\zeta=\infty$. Hence, (b) follows from (a). 
(c) If $E=[0,1]$ then (c) is a direct consequence of Theorem 2 of [2]. For $E$ being a Jordan arc, we consider a homeomorphic parametrization of $E$ by $\tau$ : $[0,1] \rightarrow E$. Since $W \circ \tau(x)$ is of bounded variation on $[0,1]$, we have, as before, that $[W \circ \tau(x), \tau(x)(W \circ \tau)(x)]=C([0,1])$. Clearly, $\tau$ induces an isometric isomorphism between $C([0,1])$ and $C(E)$. Thus, the result follows after returning to $E$ with the help of $\tau^{-1}$.

(d) is implied by Theorem 1 of [1] for $E=[0,1]$. The case of a Jordan arc can be reduced to that of the interval as in the proof of (c).

(e) First, assume that $E=\bar{D}$. Then (e) follows at once from [24, p. 135] (see also [3]). It is well known that the conformal mapping $\phi: G \rightarrow D$ extends as a diffeomorphism between $\bar{G}$ and $\bar{D}$ (with nonvanishing derivatives of $\phi$ and $\psi:=\phi^{-1}$ ), because $G$ is bounded by an analytic Jordan curve. Using $\phi$, the result for $E=\bar{G}$ is a consequence of [24, p. 135] too.

Proof of Proposition 2.6. Since $W(z)$ is a continuous nonvanishing function on $E$ and $w(z)$ of $(2.1)$ is so too, then the existence of $\mu_{w}$ and inequalities (2.4)-(2.5) follow from Theorem I.1.3 of [18]. Moreover, $W(z)$ is analytic in the interior of $E$, which implies that $S_{w} \subset \partial E$ by Theorem IV.1.10(a) of [18] and (2.1). The inequality (2.3) is a direct consequence of Theorem III.2.1 of [18].

Proof of Theorem 3.1. We have that $[W(z), z W(z)]=C(E)$ by the assumption of the theorem. Thus, $A(E, W)$ is a closed ideal of $C(E)$ (cf. Proposition 2.3), which is known to be described by its zero set (see [21, p. 32]).

Proof of Theorem 3.2. We essentially follow the proof of Theorem 2 of [10]. Suppose that there exist $f_{0} \in A(E, W)$ and $z_{0} \in E \backslash S_{w}$ such that $f_{0}\left(z_{0}\right) \neq 0$ and $W^{n} P_{n} \rightarrow f_{0}$ uniformly on $E$, as $n \rightarrow \infty$.

It is clear that $\left.f_{0}\right|_{S_{w}} \in A\left(S_{w}, W\right)$. Recall that $S_{w} \subset \partial E$ by Proposition 2.6, i.e., $S_{w}$ has empty interior. Applying Theorem 3.1, with $E$ replaced by $S_{w}$, we obtain that $A\left(S_{w}, W\right)$ is described by the zero set $Z_{W}^{*} \subset S_{w}$. Observe that multiplying $A\left(S_{w}, W\right)$ by $\left(z-z_{0}\right) W(z)$, we obtain a closed ideal of $[W(z), z W(z)]=C\left(S_{w}\right)(\mathrm{cf}$. Proposition 2.3), which consists of all functions, uniformly approximable on $S_{w}$ by the weighted polynomials $W^{n}(z) Q_{n}(z)$ such that $Q_{n}\left(z_{0}\right)=0$, as $n \rightarrow \infty$. On the other hand, the zero set of the ideal $\left(z-z_{0}\right) W(z) A\left(S_{w}, W\right)$ coincides with that of $A\left(S_{w}, W\right)$. It follows that $\left(z-z_{0}\right) W(z) A\left(S_{w}, W\right)=A\left(S_{w}, W\right)$ (see [21, p. 32]) and that $\left.f_{0}\right|_{S_{w}} \in\left(z-z_{0}\right) W(z) A\left(S_{w}, W\right)$.

Thus, there exists a sequence of the weighted polynomials $\left\{W^{n} Q_{n}\right\}_{n=0}^{\infty}$, with $Q_{n}\left(z_{0}\right)=0$, uniformly convergent to $f_{0}$ on $S_{w}$, as $n \rightarrow \infty$. Since $W^{n}(z)\left(P_{n}(z)-Q_{n}(z)\right)$ converges to zero uniformly on $S_{w}$ and converges to $f_{0}\left(z_{0}\right) \neq$ 0 for $z=z_{0} \in E \backslash S_{w}$, as $n \rightarrow \infty$, then we obtain a direct contradiction with (2.7) for some sufficiently large $n$.

Consequently, if $f \in A(E, W)$ then $f(z)=0$ for any $z \in E \backslash S_{w}$. Furthermore, the same is true for any $z \in \overline{E \backslash S_{w}}$ by the continuity of $f(z)$.

Proof of Theorem 4.1. Since $[W(z), z W(z)]=A(\bar{D})$ by the assumption of the theorem, then $A(\bar{D}, W)$ is a closed ideal of $A(\bar{D})$ by Proposition 2.3. The result now follows from the description of nontrivial closed ideals of the disk algebra (see [17] and [7, pp. 82-87]).

Proof of Theorem 4.2. First, we obtain that $A(\bar{G}, W)$ is a closed ideal of $A(\bar{G})$ (cf. Proposition 2.3). It is well known that the conformal mapping $\phi$ extends to a 
homeomorphism between $\bar{G}$ and $\bar{D}$, defining an isometric isomorphism between the algebras $A(\bar{G})$ and $A(\bar{D})$, and their closed ideals. Thus, we apply the result of [17] to the isomorphic image of $A(\bar{G}, W)$ in $A(\bar{D})$, as in the proof of Theorem 4.1, and return to $A(\bar{G}, W)$ with the help of the inverse conformal mapping $\psi$.

Proof of Theorem 4.4. Since $G$ is a Jordan domain, then the set $\left\{z:\left|z-z_{0}\right|<\right.$ $\delta, z \in \bar{G}\}$ has positive logarithmic capacity for any $z_{0} \in \bar{G}$ and $\delta>0$. It is clear that $S_{w}$ is contained in some Jordan arc, as a proper closed subset of $\partial G$, so that $\overline{\mathbb{C}} \backslash S_{w}$ is connected. Observe that all conditions of Theorem 3.2 are satisfied in this case, which yields that any function $f \in A(\bar{G}, W)$ must vanish on $\overline{\left(\bar{G} \backslash S_{w}\right)}=\bar{G}$.

\section{FurTher REMARKS}

One of the main assumptions of the theorems in Sections 3 and 4 is that $[W(z), z W(z)]=A(E)$. This equality is valid for a wide classes of sets $E$ and weights $W \in A(E)$, as described in Proposition 2.5. On the other hand, it is clear that our results may be extended further. The main ingredients of such extentions are:

(i) a proof of the equality $[W(z), z W(z)]=A(E)$;

(ii) a description of the closed ideals of $A(E)$.

Nevertheless, we believe that a problem of much greater interest and difficulty is to uncover a more explicit relation between the zero set $Z_{W}$ (or $H_{W}$ and the inner function $g_{W}$ ) and the weight $W(z)$. A considerable progress has been achieved on this problem for real valued weights on the real line (see [22], [18, Ch. VI] and [8]-[9]), but a general description of the set $Z_{W}$ through $W(z)$ is unknown even in the latter case.

\section{REFERENCES}

[1] H. Alexander, Polynomial approximation and analytic structure, Duke Math. J. 38 (1971), 123-135. MR 44:477

[2] H. Alexander, Polynomial approximation and hulls in sets of finite linear measure in $\mathbb{C}^{n}$, Amer. J. Math. 93 (1971), 65-74. MR 44:1841

[3] R. G. Blumenthal, Holomorphically closed algebras of analytic functions, Math. Scand. 34 (1974), 84-90. MR 52:1323

[4] P. B. Borwein and W. Chen, Incomplete rational approximation in the complex plane, Constr. Approx. 11 (1995), 85-106. MR 95k:41024

[5] T. W. Gamelin, Uniform Algebras, Chelsea Publ. Co., New York, 1984.

[6] T. W. Gamelin, Polynomial approximation on thin sets, Symposium on Several Complex Variables (R. M. Brooks, ed.), pp. 50-78, Lecture Notes in Math., vol. 184, Springer-Verlag, Berlin, 1971. MR 45:9145

[7] K. Hoffman, Banach spaces of analytic functions, Prentice-Hall, Englewood Cliffs, 1962. MR 24:A2844

[8] A. B. J. Kuijlaars, The role of the endpoint in weighted polynomial approximation with varying weights, Constr. Approx. 12 (1996), 287-301.

[9] A. B. J. Kuijlaars, Weighted approximation with varying weights: the case of a power type singularity, J. Math. Anal. Appl. 204 (1996), 409-418. CMP 97:04

[10] A. B. J. Kuijlaars, A note on weighted polynomial approximation with varying weights, J. Approx. Theory 87 (1996), 112-115. CMP 97:01

[11] K. Kuratowski, Topology, vol. II, Academic Press, New York, 1968.

$[12]$ G. G. Lorentz, Approximation by incomplete polynomials (problems and results), Padé and Rational Approximations: Theory and Applications (E. B. Saff and R. S. Varga, eds.), pp. 289-302, Academic Press, New York, 1977. MR 57:6956

[13] D. S. Lubinsky and E. B. Saff, Uniform and mean approximation by certain weighted polynomials, with applications, Constr. Approx. 4 (1988), 21-64. MR 88j:41049 
[14] D. S. Lubinsky and V. Totik, Weighted polynomial approximation with Freud weights, Constr. Approx. 10 (1994), 301-315. MR 95i:41007

[15] I. E. Pritsker and R. S. Varga, The Szegö curve, zero distribution and weighted approximation, Trans. Amer. Math. Soc. 349 (1997), 4085-4105. CMP 96:17

[16] I. E. Pritsker and R. S. Varga, Weighted polynomial approximation in the complex plane, Electron. Res. Announc. Amer. Math. Soc. 3 (1997), 38-44. CMP 97:11

[17] W. Rudin, The closed ideals in an algebra of analytic functions, Can. J. Math. 9 (1957), 426-434. MR 19:641c

[18] E. B. Saff and V. Totik, Logarithmic Potentials with External Fields, Springer-Verlag, Heidelberg, 1997.

[19] E. B. Saff and R. S. Varga, On incomplete polynomials, Numerische Methoden der Approximationstheorie (L. Collatz, G. Meinardus and H. Werner, eds.), ISNM 42, pp. 281-298, Birkhäuser-Verlag, Basel, 1978. MR 80d:41008

[20] N. Sibony and J. Wermer, Generators for $A(\Omega)$, Trans. Amer. Math. Soc., 194 (1974), 103114. MR 54:7856

[21] E. L. Stout, The Theory of Uniform Algebras, Bogden and Quigley, Inc., Belmont, 1971. MR 54:11066

[22] V. Totik, Weighted Approximation with Varying Weights, Lecture Notes in Math., vol. 1569, Springer-Verlag, Heidelberg, 1994. MR 96f: 41002

[23] M. Tsuji, Potential Theory in Modern Function Theory, Maruzen, Tokyo, 1959. MR 22:5712

[24] J. Wermer, Banach Algebras and Several Complex Variables, Springer-Verlag, New York, 1976. MR 52:15021

Institute for Computational Mathematics, Department of Mathematics and ComPuter Science, Kent State University, Kent, Ohio 44242-0001

E-mail address: pritsker@mcs.kent.edu

Current address: Department of Mathematics, Case Western Reserve University, 10900 Euclid Avenue, Cleveland, Ohio 44106-7058

E-mail address: iep@po.cwru.edu 